\title{
Validation of SWMF magnetic field and plasma
}

\author{
D. T. Welling ${ }^{1}$ and A. J. Ridley ${ }^{1}$
}

Received 11 May 2009; revised 18 November 2009; accepted 19 November 2009; published 19 March 2010.

[1] The Space Weather Modeling Framework (SWMF), using the coupled BATSRUS model, the Rice Convection Model, and an ionosphere electrodynamics solver, is used to simulate 10 space weather events. The simulations are completed in near real time using a limited amount of computational resources. Satellite-specific magnetic field, plasma energy-density spectra, and integrated particle density and temperature are compared against in situ measurements to validate the coupled system. Results are examined both qualitatively and quantitatively to assess model performance. The results show that the system is adept at reproducing large-scale magnetic field variations and predicts plasma temperature and density at values near to the measured mean. Smaller-scale features, such as dipolarizations of the magnetic field at geosynchronous locations, are not reproduced well. Lack of cold particle sources in the inner magnetosphere causes the particle results to suffer. This work is part of a larger effort to thoroughly validate the SWMF.

Citation: Welling, D. T., and A. J. Ridley (2010), Validation of SWMF magnetic field and plasma, Space Weather, 8, S03002, doi:10.1029/2009SW000494.

\section{Introduction}

[2] Numerical modeling of the near-Earth space environment has become a broad and important field. Models range from empirical codes [Papitashvili and Rich, 2002; Tsyganenko, 2002a, 2002b; Weimer, 1996] to large, first principle based systems [Powell et al., 1999; Gombosi et al., 2002; Raeder et al., 2001; Lyon et al., 2004]. These codes are invaluable tools for learning more about the near-Earth environment. Because space weather conditions can impact communications, ground based utilities, and even airlines [Baker, 2000; Feynman and Gabriel, 2000; Horne, 2001; Pirjola et al., 2005], numerical models of the space environment are becoming as equally vital as operational forecast tools as they are scientific tools.

[3] The University of Michigan's Center for Space Environment Modeling (CSEM) has recently developed the Space Weather Modeling Framework (SWMF), a flexible system that allows many models of the space environment to be run concurrently and coupled together easily [Tóth et al., 2005]. It accomplishes this by dividing the Sun to upper atmosphere system into 11 physical components or "modules." Each module contains a numerical model whose execution is controlled by the SWMF in order to synchronize the entire system. Coupling between modules through the SWMF is configured to simulate the interactions between the different physical systems. The framework has been used to perform complicated studies of

\footnotetext{
${ }^{1}$ Department of Atmospheric, Oceanic, and Space Sciences, University of Michigan, Ann Arbor, Michigan, USA.
}

the Sun to Earth system that are easily performed by several coupled models working together [Tóth et al., 2007; Ridley, 2007].

[4] As models mature, it becomes increasingly necessary to validate the results against measurements of the environment being modeled. Thorough validation expands the understanding of models and aids in the interpreting of their results. It also allows code developers to track the impact that changes in the code have on the results. Validation is necessary for operational applications of numerical models, as users rely on the code's accuracy.

[5] This paper presents validation results of the SWMF, using three components, against in situ magnetic field and plasma measurements. The validation is performed over a wide range of magnetospheric conditions, and the simulations are run in the same manner as they would under operational conditions. Performance of the SWMF to predict these measurements is assessed both qualitatively and quantitatively. These results will serve as a baseline to compare to future validation as the SWMF's capabilities are expanded.

[6] To increase the operational relevance of this study, the system's capability to predict satellite crossings of the magnetopause at geosynchronous orbit is investigated. These events cause a rapid change in the near-spacecraft plasma environment and can trigger a spacecraft anomaly. Prediction of such crossings would help fulfill the list of user requirements listed in the European Space Agency's 2001 survey [Horne, 2001].

[7] This work is the first intensive validation of magnetospheric magnetic field and plasma properties as pre- 
Table 1. List of Events Chosen for This Study With Start, Duration, and a Short Description

\begin{tabular}{lccc}
\hline \multicolumn{1}{c}{ Event Date } & $\begin{array}{c}\text { Start Time } \\
\text { (UT) }\end{array}$ & $\begin{array}{c}\text { Duration } \\
(\mathrm{h})\end{array}$ & Quick Description \\
\hline 9 December 1996 & 1800 & 30 & high-speed stream \\
4 May 1998 & 0200 & 12 & strongly driven storm \\
15 July 2000 & 1400 & 12 & Bastille Day event \\
31 March 2001 & 0200 & 12 & CME-driven storm \\
4 August 2001 & 1200 & 12 & steady Bz IMF \\
31 August 2001 & 1200 & 12 & pressure triggered substorm \\
17 April 2002 & 0800 & 24 & sawtooth storm \\
29 October 2003 & 0200 & 28 & first Halloween CME \\
20 November 2003 & 0400 & 20 & strong storm \\
2 September 2004 & 1600 & 6 & untriggered substorm \\
\hline
\end{tabular}

dicted by the SWMF when using the coupled models described below. Each module has been validated individually to varying degrees [Powell et al., 1999; Ridley et al., 2001; Tóth et al., 2007], and limited data-model comparisons of the system have been made in the past [Ridley et al., 2002]. This study expands upon this previous work and is part of a larger validation effort of the SWMF, whose results are described by $Y u$ and Ridley [2008] and Wang et al. [2008].

\section{Procedure}

[8] Ten events, listed in Table 1, were selected for simulation. The events were selected to provide a broad range of space weather conditions. Each event is simulated using the Space Weather Modeling Framework (SWMF) with three physical modules activated (details below). Satellitespecific magnetic field and particle data is extracted to make one to one comparisons with several different scientific satellites. To further demonstrate the operational capabilities of the system, magnetopause crossings of geosynchronous satellites are treated as binary events, with contingency tables constructed to quantify predictive performance of these events.

\subsection{Model Description and Setup}

\subsubsection{SWMF}

[9] In this study, the SWMF was configured to use three components: Global Magnetosphere (GM), Inner Magnetosphere (IM), and Ionospheric Electrodynamics (IE). The system is configured to achieve near real time run speeds using 32 processors on NASA's "Columbia" SGI Altix machine. Simulation parameters are constant throughout the study; inputs and parameters are not tailored to individual events. Coupling of the components occurs every $5 \mathrm{~s}$ of simulation time for GM-IM and every $10 \mathrm{~s}$ for GM-IE and IM-IE.

\subsubsection{Global Magnetosphere}

[10] The GM component simulates the magnetosphere, from the bow shock to the distant tail. It is responsible for processes such as the transfer of energy and mass from the solar wind to the magnetosphere and magnetospheric convection. It provides the IE module with the field aligned current location and strength and the IM module with magnetic field structure and plasma density and temperature at the IM boundary [De Zeeuw et al., 2004].

[11] The GM component is modeled by the single fluid version of the Block Adaptive Tree Solar wind Roe-type Upwind Scheme (BATSRUS) code. BATSRUS is detailed by Powell et al. [1999] and De Zeeuw et al. [2000]. It solves the three-dimensional ideal magnetohydrodynamic (MHD) equations for mass, momentum, and energy density as well as magnetic field. It uses an adaptive Cartesian grid in Geocentric Solar Magnetospheric (GSM) coordinates. The inner boundary is a sphere of radius 2.5 Earth radii $\left(R_{E}\right)$; the outer boundary is $32 R_{E}$ in the upstream direction, $224 R_{E}$ downstream, and $128 R_{E}$ in each other direction. Observed upstream solar wind conditions (either from the ACE or Wind spacecraft measurements) are imposed as the upstream boundary conditions.

[12] The resolution in this study is coarser than what is typically used for scientific studies in order to achieve near real time simulation completion. Toward the inner boundary, the resolution is the finest $\left(1 / 4 R_{E}\right)$. Cell size increases to $1 / 2 R_{E}$ at geosynchronous locations and to $1 R_{E}$ for the outer magnetosphere and plasma sheet region. This grows, by factors of 2 , to a maximum cell size of $8 R_{E}$.

[13] The BATSRUS model provides the magnetic field values that are compared against in situ measurements. To simplify the comparison, "virtual satellites" are flown in BATSRUS. Satellite orbits are given to the code as part of the input. Then, as the event is simulated, the MHD solution is interpolated to the satellite's position and saved. In this way, one-to-one comparisons of measured and predicted values can be made easily.

\subsubsection{Inner Magnetosphere}

[14] The inner magnetosphere domain is the region that is characterized by closed magnetic field lines and particles of $\mathrm{keV}$ energies. To simulate this region, the Rice Convection Model (RCM) [Harel et al., 1981; Sazykin, 2000; Toffoletto et al., 2003] is used. This model treats the inner magnetosphere plasma as an isotropic, slowly flowing ( $V \ll V_{\text {thermal }}$ and $V \ll V_{\text {Alfuen }}$ [Wolf, 1983]) fluid that is electromagnetically drifting due to corotation and electromagnetic fields. The plasma is divided into many independent populations of varying energy and species, whose flux tube averaged characteristics are advected through the magnetosphere. The IM component receives flux tube volumes from the GM component and returns pressure values to correct those calculated in GM [De Zeeuw et al., 2004]. It receives the ionospheric electric potential from the IE component. The density and temperature initial and boundary values are computed from the GM solution.

[15] Because the IM component yields more complete information about the inner magnetosphere particle distribution than the other components used, its solution is used for the plasma density and temperature comparisons. Extraction of this information along a satellite orbit, as done in BATSRUS, is more complicated because of 

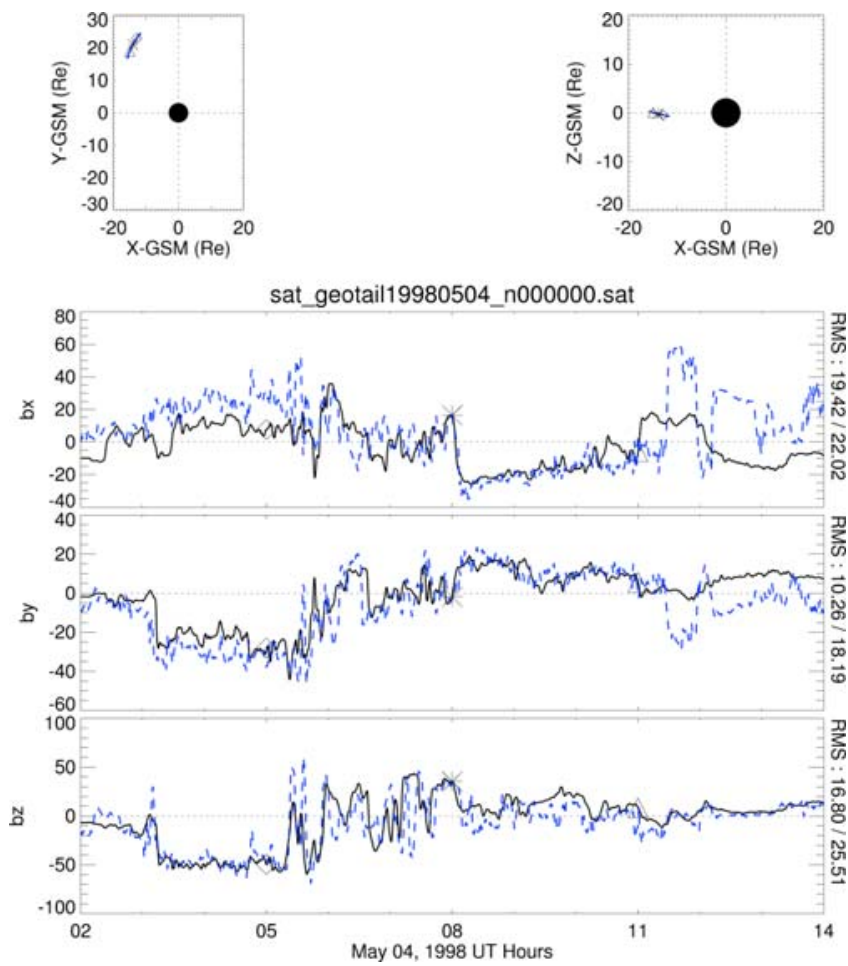

Figure 1. Comparison between Geotail measured (blue dashed lines) and SWMF forecasted (black solid lines) magnetic field (nT) in three orthogonal components in GSM coordinates. The satellite's position during the event is displayed in the top two plots, with the star, diamond, and triangle symbols used on both the orbit and magnetic field plots to help coordinate the two.

RCM's 2-D ionospheric grid. To determine a satellite's position on this grid, the magnetic field line passing through the satellite's location is traced down to the ionosphere. This tracing is performed by the BATSRUS code (detailed in the work by De Zeeuw et al. [2004]), and the ionospheric footprint of the satellite is passed to RCM when the codes are coupled together. The RCM solution is then interpolated to this position to provide satellitespecific particle distribution information.

2.1.4. Ionospheric Electrodynamics

[16] The IE component calculates height integrated ionospheric quantities at an altitude of about $110 \mathrm{~km}$. It currently is handled by the ionosphere electrodynamics solver described by Ridley and Liemohn [2002] and Ridley et al. [2004]. This model receives field-aligned currents from the GM component and uses them to calculate particle precipitation and conductance patterns. The conductance and field-aligned currents are used to calculate the electric potential, which is in turn mapped back to the inner boundary of the GM module. The ionospheric potential is also used by the IM component as described above.

\subsection{Data-Model Comparisons}

[17] Satellite specific data, extracted as described by the GM and IM modules, is compared to several in situ sources to assess model performance. Magnetic field results are compared to fluxgate magnetometer measurements aboard the Polar, Geotail, GOES 8 to GOES 12, Wind, and Cluster satellites. Particle density and energy results from the RCM model are converted to energy flux and compared to measured spin-averaged energy flux values from the Magnetospheric Particle Analyzer (MPA) instrument aboard the LANL geosynchronous spacecrafts. The instrument and data are described by McComas et al. [1993]. Quantitative assessments of plasma density and temperature are made by comparing to density and temperature moments of the MPA measured distribution function for $\sim 100 \mathrm{eV}$ to $40 \mathrm{keV}$ (ions) and $\sim 30 \mathrm{eV}$ to $40 \mathrm{keV}$ (electrons) energy windows. RCM results are integrated over equivalent energy ranges to ensure proper comparisons.

[18] Two mathematical tools are used to quantify the SWMF performance: normalized root mean squared error (nRMSE) and correlation coefficient. Correlation coefficient is discussed further by Jolliffe and Stephenson [2003], while nRMSE is also applied in the work by Ridley et al. [2002], Yu and Ridley [2008], and Wang et al. [2008]. The correlation coefficient ranges from -1 (data and model are anticorrelated) to +1 (perfect correlation), with 0 indicating
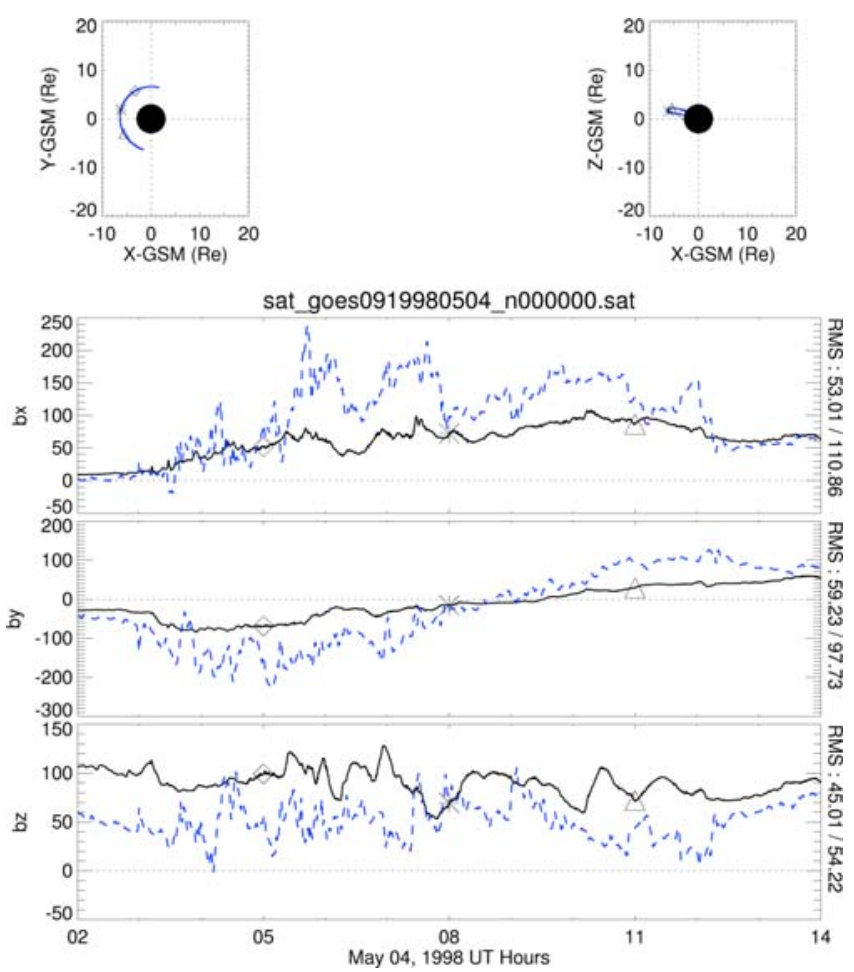

Figure 2. Similar to Figure 1 but for the GOES 9 geosynchronous satellite. 


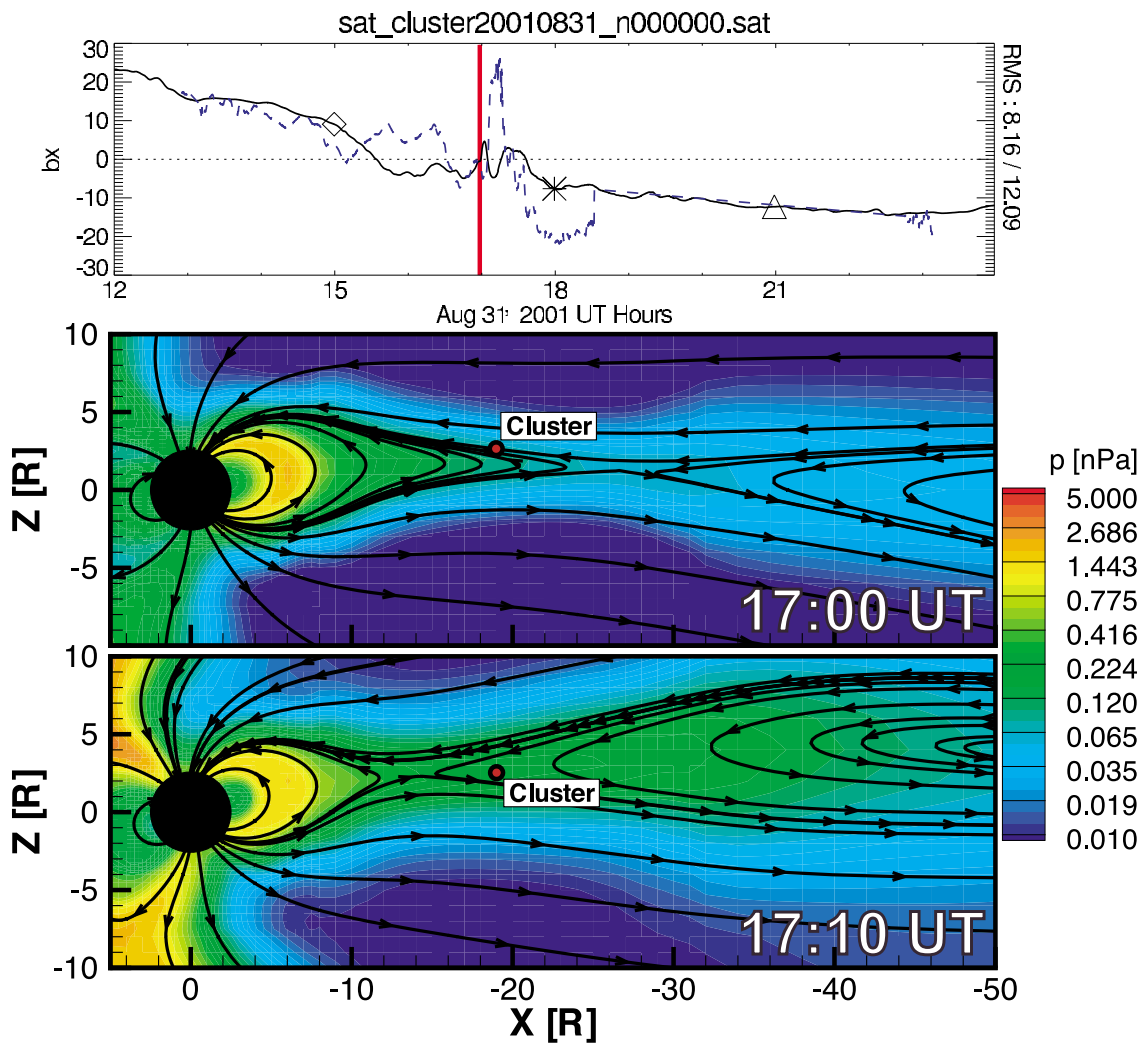

Figure 3. Results from the 31 August 2001 event, where an observed substorm is reproduced by the coupled models. (top) The model (solid black) and data (blue dashed) comparison of the $X$ component of the magnetic field for a single Cluster satellite. The vertical red line marks 1700 UT. (middle and bottom) Shown are $Y=0$ slices of the magnetotail taken from the simulation results. Field lines are traced in black, the contours show pressure $(\mathrm{nPa})$, and the red dot indicates the location of the Cluster satellite used in the top plot. At $1700 \mathrm{UT}$, the plasma sheet is thin and elongated before a plasmoid quickly forms and travels downtail. At 1710 UT (bottom plot), the plasmoid has passed the Cluster constellation. This behavior is reflected in both the measured and modeled data shown in the top plot.

no correlation. nRMSE ranges from 0 (model is perfect prediction of data) to $\infty$. A value of 1 indicates that the predicted values are similar to predicting a constant value of zero. The formulas for both metrics are presented in equations (1) and (2), where $x$ is the measured value, $y$ is the predicted value, and $n$ is the number of data-model pairs used in the calculation.

$$
\begin{gathered}
\text { Corr. Coeff. }=\sqrt{\frac{\left(\sum_{i}^{n} x_{i} y_{i}-n \overline{x y}\right)^{2}}{\left(\sum_{i}^{n} x_{i}^{2}-n \bar{x}\right)\left(\sum_{i}^{n} y_{i}^{2}-n \bar{y}\right)}} \\
n R M S E=\sqrt{\frac{\sum_{i}^{n}\left(x_{i}-y_{i}\right)^{2}}{\sum_{i}^{n} x_{i}^{2}}}
\end{gathered}
$$

\subsection{Magnetopause Crossings}

[19] At geosynchronous orbit, magnetopause crossings can be detected in both magnetic field and particle data. In magnetic field data, a sudden change from magnetospheric field to solar wind field (typically, a sharp drop in $\mathrm{Bz}$ in GSM coordinates) indicates a crossing [Rufenach et al., 1989]. In MPA spectrograms, a sudden change in the particle distribution is observed as the spacecraft moves from the magnetosphere to the magnetosheath [McComas et al., 1994]. In RCM data, a crossing appears as a period of missing data because the spacecraft no longer resides in the RCM domain (closed field lines.) All crossings captured in the model are due to BATSRUS results, as this model provides the magnetic field configuration for RCM.

[20] Magnetopause crossings are treated as binary events where a useful forecast would be an unqualified statement that the event will or will not happen. A discussion on the handling of binary events can be found in 

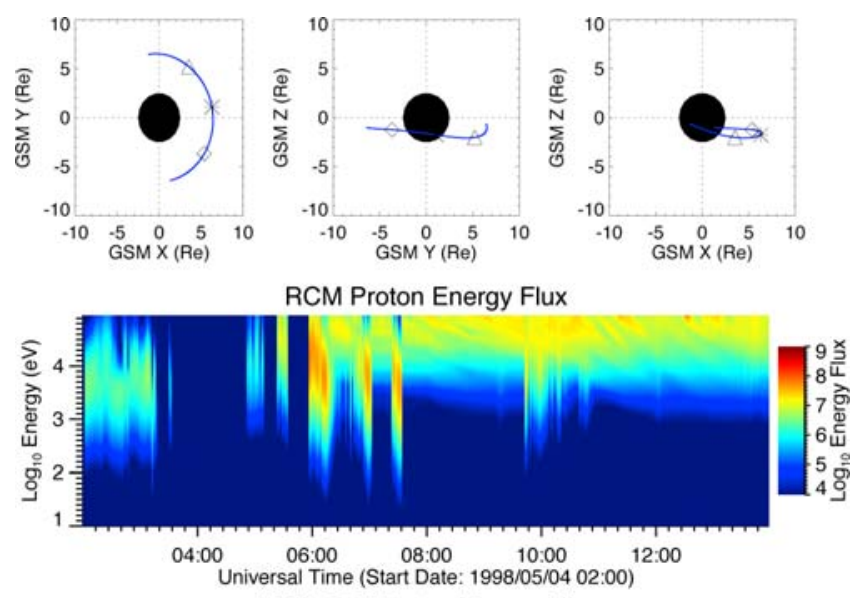

LANL-97A Proton Energy Flux

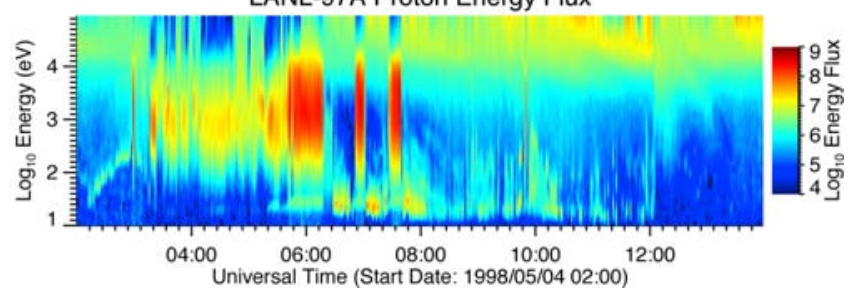

Figure 4. (top) The position of the LANL 97A geosynchronous satellite in three planes (GSM coordinates). Proton energy flux comparison for the 4 May 1998 event at the LANL 97A geosynchronous satellite, (middle) as simulated by the coupled codes and (bottom) as measured by the LANL MPA instrument. The color bar is in units of energy flux $\log _{10}\left(\mathrm{eV} \mathrm{cm}^{-2} \mathrm{~s}^{-1} \mathrm{str}^{-1} \mathrm{eV}^{-1}\right)$.

the work by Jolliffe and Stephenson [2003]. The ability of the coupled system to predict these crossings is examined on two time scales: whole event and hourly. For each time increment, there are four possible outcomes: a "hit" (event was predicted and occurred), a "miss" (event occurred but was not predicted), a "false alarm" (event was predicted but did not occur), and a "true negative" (event was neither predicted or occurred.) The results are tallied into contingency Tables 4 and 5, and several basic metrics are calculated: probability of detection ( $\mathrm{POD}$, hits divided by number of observed crossings), false alarm rate (number of false alarms divided by total number of nonoccurrences), and critical success index (CSI) (number of hits divided by total number of occurrences plus false alarms). CSI is especially suited for scoring predictions of rare events because it neglects nonoccurrences. The Heidke skill score (HSS), shown in equation (3), is also used to measure performance. For this calculation, $P C$ is the proportion of correct forecasts to total number of intervals while $E$ is the proportion correct if the forecasts and observations were truly independent and assuming the same proportion of forecasts of occurrences to nonoccurrences. An HSS of 1 is a perfect score while an HSS of zero indicates no improvement compared to a random forecast.

$$
H S S=\frac{P C-E}{1-E}
$$

\section{Analysis and Discussion}

[21] Figure 1 displays the data-model comparison for the Geotail satellite during the 4 May 1998 event. The top plots of Figure 1 show the satellite's position during the event in the $X-Y$ and $X-Z$ planes. The second, third, and fourth plots of Figure 1 show the measured (blue dashed) and forecast (black solid) magnetic field in three components. During this event, the Geotail satellite is residing in the dusk flank magnetosheath region. As demonstrated by the data-model comparison, BATSRUS does a good job at folding the Interplanetary Magnetic Field (IMF) through

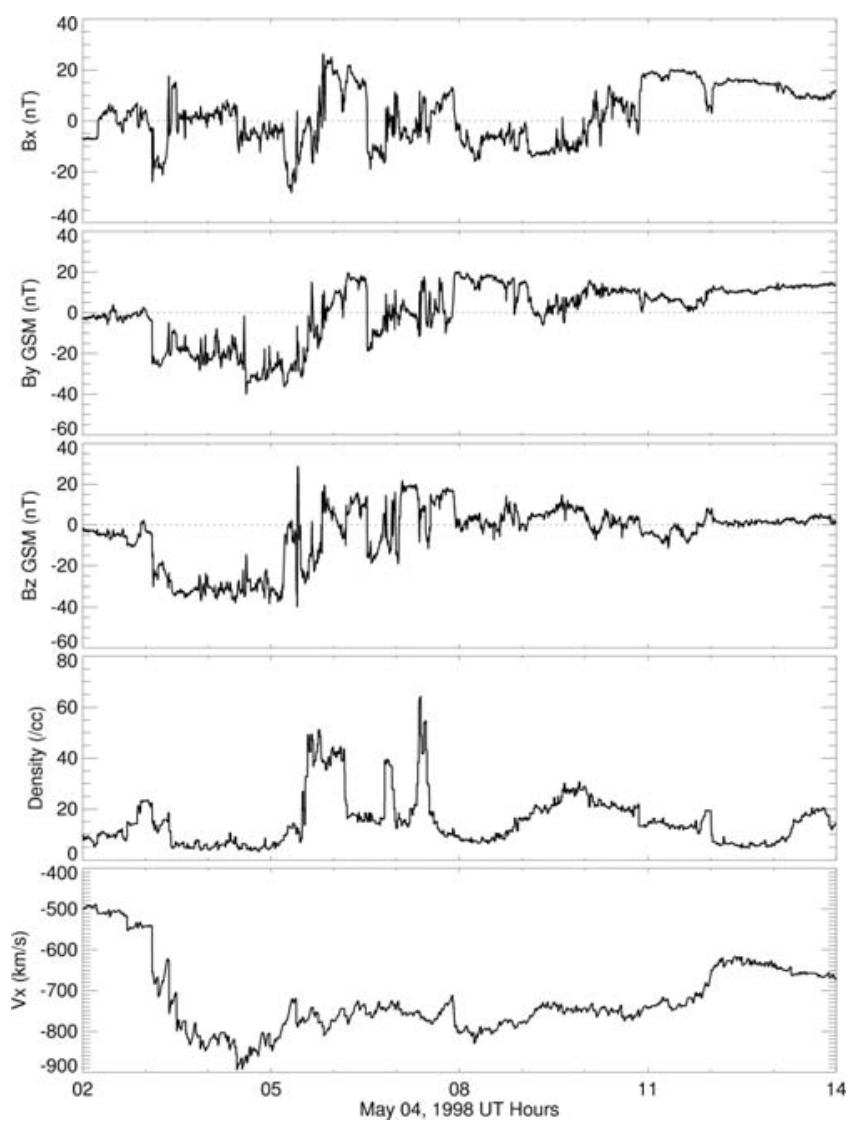

Figure 5. Solar wind drivers during the 4 May 1998 event as measured by the ACE spacecraft. Shown, from top to bottom, are the $X, Y$, and $Z$ components of the IMF (nT); proton number density $\left(1 / \mathrm{cm}^{3}\right)$; and Earthward flow velocity $(\mathrm{km} / \mathrm{s})$. 

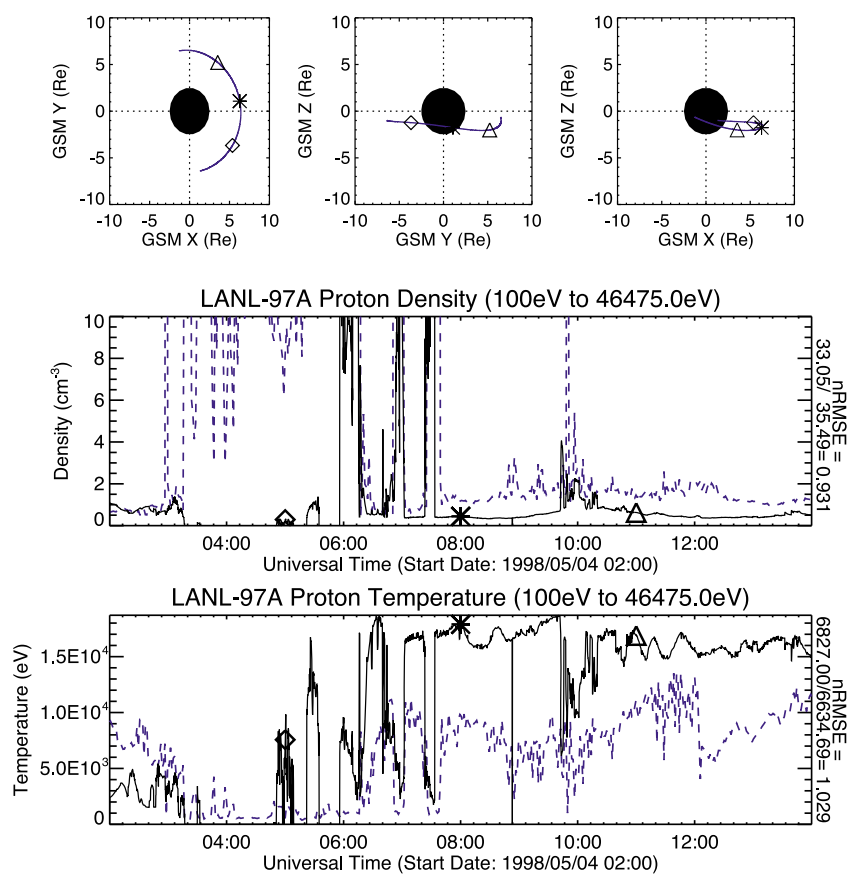

Figure 6. A data-model comparison similar to Figure 2 but for integrated proton temperature and density at the LANL 97A satellite. The simulation result (black line) was obtained by integrating the RCM result from Figure 4 over the same energy window that is used to generate the LANL MPA moment data (blue dashed line). Typical of results throughout the study, RCM plasma for this energy range is too warm and less dense than the in situ measurement.

the bow shock and around the magnetopause. Toward the end of the event, the differences between the data and the model are due to discrepancies in the location of the magnetopause. With an extremely coarse grid at this location, such a discrepancy is not surprising. This comparison is a typical example of outer magnetosphere results.

[22] Similar to Figure 1, Figure 2 displays the data-model comparison for the GOES 9 geosynchronous satellite during the 4 May 1998 event. Typical of all geosynchronous magnetic field comparisons in this study, the largescale features of the magnetic field are captured well. However, the $\mathrm{Z}$ component is consistently over predicted, while the $X$ and $Y$ components are consistently under predicted. This demonstrates that the SWMF magnetic field is less stretched than the measured field. Remedying this issue requires a stronger ring current. Despite this shortcoming, the metric scores are still favorable (as discussed in section 4).

[23] Two dipolarizations of the magnetic field, caused by events such as substorms and sawteeth, are observed in the $\mathrm{Z}$ component of the measured data at 0800 and 0900 universal time (UT). Small time and spatial features such as these are nearly always missed, but do not hamper the metrics significantly because of their brevity. Although the geosynchronous results would imply that the coupled codes are incapable of reproducing such events, this is not the case. Figure 3 displays results from the 31 August 2001 event, where a plasmoid forms and releases both in the modeled and observed magnetotail. Figure 3 (top) shows the $\mathrm{X}$ component of the magnetic field as measured by a single Cluster satellite (blue dashed line) and as predicted by the SWMF (black line). The middle and bottom plots of Figure 3 show $Y=0$ slices of the magnetotail. Field lines are traced in black; the contours show pressure. At 1700 UT (Figure 3, middle), the plasma sheet is thin and elongated before a plasmoid quickly forms and travels down tail. At 1710 (Figure 3, bottom), the plasmoid has passed the cluster constellation. This behavior is reflected in both the measured and modeled magnetic field in Figure 3 (top). The differences between the two indicate a difference in plasmoid size and location, but the timing of the event is correct.

[24] While substorms such as the one observed in Figure 3 form frequently in the model results, they are not observed in the model results at geosynchronous orbit. An explanation for this may be that the reconnection rate in the tail is too fast, thus not allowing for enough energy to

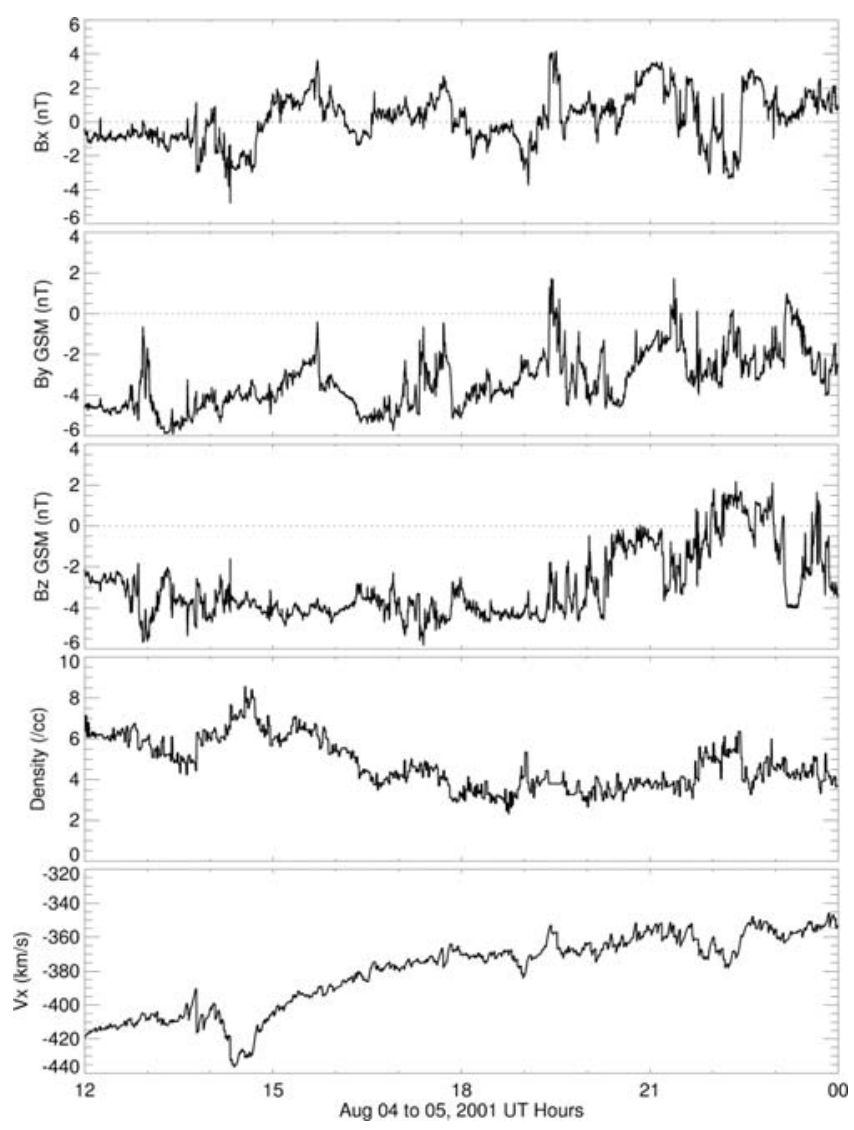

Figure 7. Same as Figure 5 but for the 4 August 2001 event. 


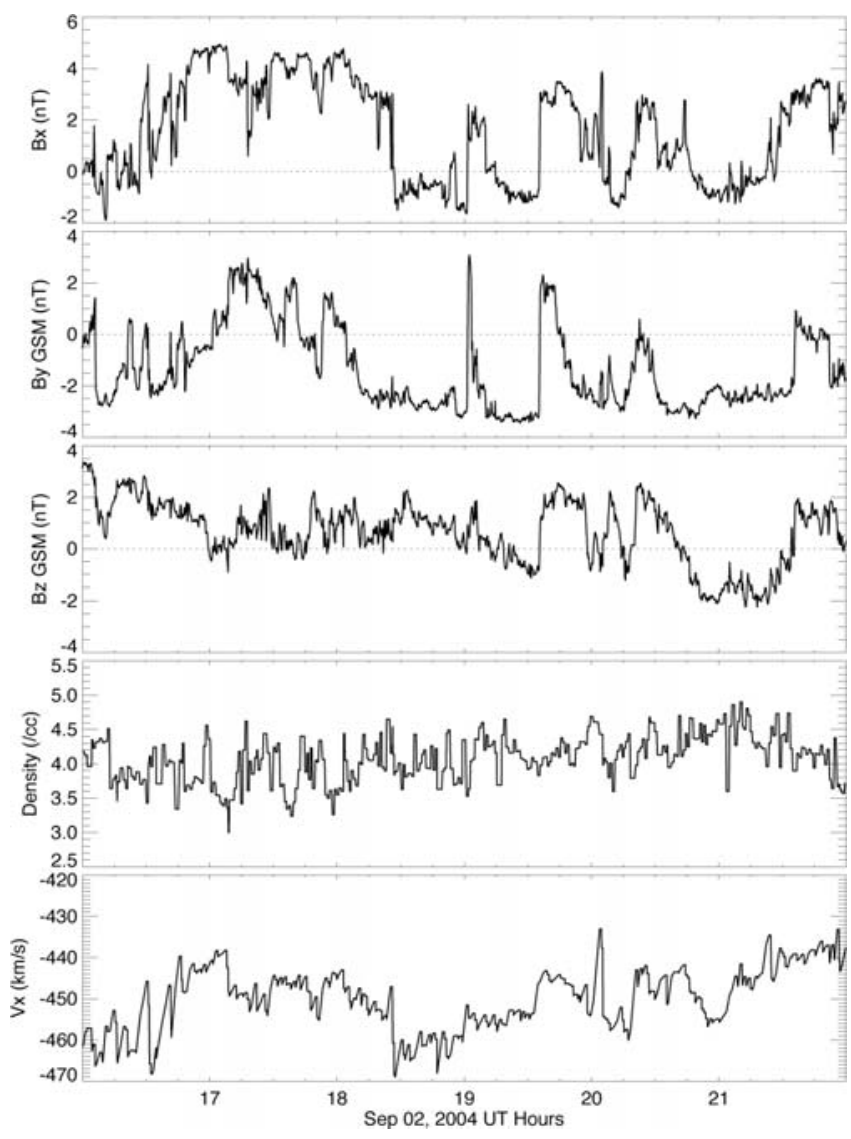

Figure 8. Same as Figure 5 but for the 2 September 2004 event.

build up in the tail before the plasmoid is released. This hypothesis requires further investigation, which, using the events selected for this study, is hampered by limited data coverage in the tail region.

[25] Examining magnetopause crossings may provide further information about the above issues. Figure 4 displays the proton results from the LANL 97A satellite during the 4 May 1998 event. The top plots of Figure 4 show the position of the satellite in three planes (GSM coordinates). During the event, the satellite spent a significant time on the dayside of the magnetosphere. Also displayed is proton energy flux $\left(\log _{10}\left(\mathrm{eV} \mathrm{cm}^{-2} \mathrm{~s}^{-1} \mathrm{str}^{-1}\right.\right.$ $\left.\mathrm{eV}^{-1}\right)$ ) as simulated by the coupled codes (Figure 4, middle) and as measured by the LANL MPA instrument (Figure 4, bottom). Several distinct magnetopause crossings are observed in the in situ measurement: a persistent crossing from approximately 0300 to $0530 \mathrm{UT}$, followed by three short, well-pronounced crossings starting at 0545 UT. The SWMF correctly predicts the first, prolonged crossing as indicated by the drop out in the RCM results. The next three, however, are not captured. During these periods, the virtual satellite is so close to the RCM outer boundary that the recorded distribution is boundary plasma provided by BATSRUS, but the magnetopause is not crossed. Figure 5 displays the solar wind drivers for this event, originally measured by the ACE spacecraft and time shifted from the Lagrangian point to the upstream boundary of the GM component. The IMF was strongly southward during the first magnetopause crossing observed in Figure 4. This crossing was clearly driven by magnetic erosion of the dayside magnetosphere. The next three crossings correspond to strong pressure pulses in the solar wind, as seen in the number density in Figure 5 (fourth plot). The model's increased sensitivity to dayside reconnection over pressure pulses as drivers for magnetopause crossings, a consistent pattern in the results, supports the conclusion that the reconnection rate in the code may be faster than the real world rate.

[26] There are numerous other factors that affect the magnetopause crossing results. Although the BATSRUS model takes into account the tilt of the Earth's intrinsic dipole field, it neglects the offset from the center of the Earth. This has the effect of artificially strengthening the field on the American sector of the Earth and weakening it on the opposite side. The low resolution used, chosen to allow the simulations to finish quickly, also plays a role. At $1 / 2 R_{E}$ cell size, one grid point can be the difference between well inside or outside of the magnetosphere. This is seen in Figure 4, where the satellite is a single cell away from being in the magnetosheath. Although these near misses stand out qualitatively, they are counted as misses in
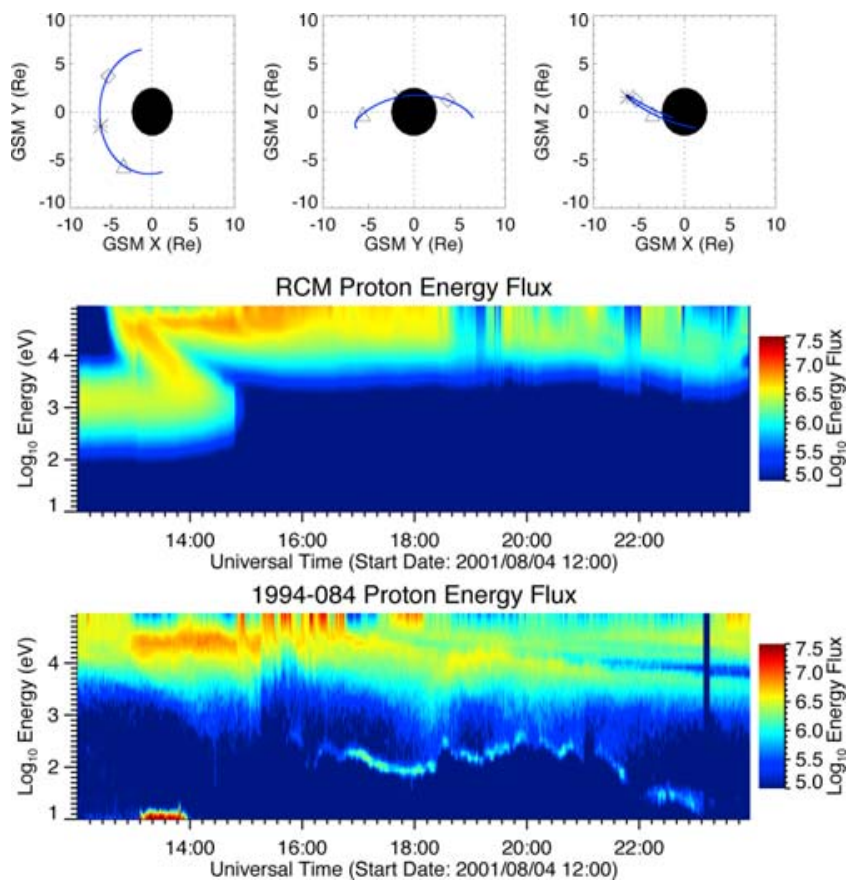

Figure 9. Similar to Figure 4 but for the LANL 1994084 satellite during the 4 August 2001 event. The modeled particle distribution is quickly accelerated to resemble the measured distribution due to the southward IMF imposed during the event. 

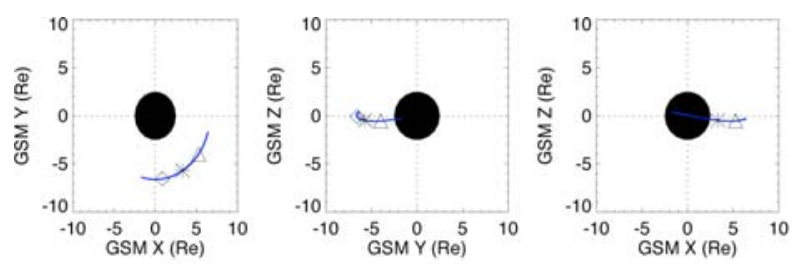

RCM Proton Energy Flux

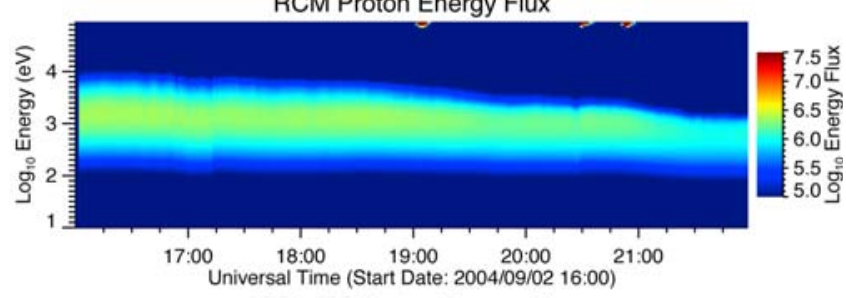

1991-080 Proton Energy Flux

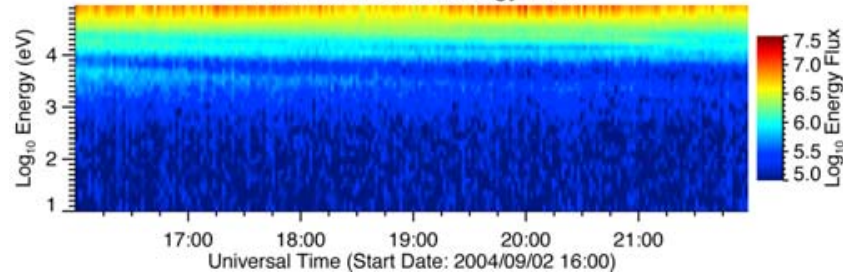

Figure 10. Proton energy flux comparison for the 2 September 2004 event. While both distributions show little evolution throughout the event, the model population, which changes little from the initial MHD Maxwellian, poorly resembles the observations.

the magnetopause crossing contingency Tables 4 and 5 discussed in section 4 .

[27] Figure 4 exemplifies the model's particle performance during storm time conditions. Because the RCM is initialized with single-fluid MHD plasma, during the first moments of the simulation, the simulated plasma distribution at all locations is a Maxwellian determined from the BATSRUS solution. Southward IMF imposes a convection electric field capable of drawing in fresh plasma from the tail boundary of the RCM, which is quickly accelerated to $\mathrm{keV}$ energies and begins to circulate around the Earth. This creates a more favorable data-model comparison of the warmer ( $\mathrm{keV}$ energies) plasma, as is observed starting at 0800 UT in Figure 4. Any southward turning leads to a fresh injection of plasma from the tail
Table 3. Average Performance Measures of Forecasted and Measured In Situ Particle Properties ${ }^{\mathrm{a}}$

\begin{tabular}{lccccc}
\hline & \multicolumn{2}{c}{$\mathrm{H}^{+}(>100 \mathrm{eV})$} & & \multicolumn{2}{c}{$e^{-}(>30 \mathrm{eV})$} \\
\cline { 2 - 3 } \cline { 5 - 6 } & Density & Temperature & & Density & Temperature \\
\hline nRMSE & 0.961 & 0.990 & & 1.096 & 1.208 \\
Correlation coefficient & 0.274 & 0.177 & & 0.266 & 0.096 \\
\hline
\end{tabular}

${ }^{a}$ Nineteen comparisons were made from the 10 events studied. All particle data was taken from the MPA instruments aboard the LANL geosynchronous satellites. Density and temperature from the model are taken from an energy range that matches the range of the MPA instrument used in the comparison.

and a corresponding dispersion pattern in the RCM results (e.g., 0800 UT, 0900 UT, 1000 UT, etc. in Figures 4 and 5). This pattern is observed in the in situ data for longer, stronger southward IMF turnings (e.g., 1045 UT), but overall dynamics are not captured accurately. There is a clear void in the simulated cold plasma distribution due to the lack of cold, ionospheric sources of particles in the version of RCM used in this study.

[28] Figure 6 shows the results from Figure 4 integrated over the $100 \mathrm{eV}$ to $46.5 \mathrm{keV}$ energy window to yield a single proton density and temperature. The effects described above are clearly observed in the integrated results. Notably, the lack of a direct cold particle source from the ionosphere into the RCM drives up the predicted proton temperature while keeping the predicted density too low. After 0800 UT, dynamics are captured poorly. These are persistent features of the data-model comparisons and are reflected in the statistics outlined in section 4.

[29] Results from the 4 August 2001 and 2 September 2004 event demonstrate the importance of IMF $B_{z}$ in the particle results. The driving solar wind conditions for the 2001 event are displayed in Figure 7. The IMF $B_{z}$ is steadily southward for the greater portion of the event (third plot), while the density and velocity remain relatively steady throughout. The solar wind drivers for the 2004 event (Figure 8) are similar, but with $B_{z}$ northward. Data-model proton energy flux comparisons are shown in Figures 9 and 10. As during the 1998 event results, both RCM particle distributions are initialized as Maxwellians using the MHD solution. The model results from the 2001 event (Figure 9) are quickly energized with fresh plasma that has been accelerated through the plasma sheet and into the

Table 2. Average Performance Measures of In Situ Magnetic Field Separated by Orbit Type

\begin{tabular}{|c|c|c|c|c|c|c|c|c|c|}
\hline \multirow[b]{2}{*}{ Orbit Type } & \multicolumn{4}{|c|}{ nRMSE } & \multicolumn{4}{|c|}{ Correlation Coefficient } & \multirow[b]{2}{*}{$\mathrm{n}$} \\
\hline & $\mathrm{Bx}$ & By & $\mathrm{Bz}$ & $|B|$ & $\mathrm{Bx}$ & By & $\mathrm{Bz}$ & $|B|$ & \\
\hline Polar & 0.362 & 0.339 & 0.343 & 0.156 & 0.947 & 0.931 & 0.941 & 0.980 & 9 \\
\hline
\end{tabular}

\footnotetext{
${ }^{a}$ Listed are the normalized root mean square errors (nRMSE) and correlation coefficients between the forecast and measured value of the three components of the magnetic field (in GSM coordinates) and the total field magnitude. The value $\mathrm{n}$ is the number of comparisons used to generate the average. A data-model pair was discarded if there was little data available or if the measurement was made upstream of the magnetosphere.
} 
Table 4. Contingency Table for Magnetopause Crossings Using Whole-Event Time Resolution ${ }^{\mathrm{a}}$

\begin{tabular}{ccc}
\hline & \multicolumn{2}{c}{ Observation } \\
\cline { 2 - 3 } Forecast & Yes & No \\
\hline Yes & 13 & 0 \\
No & 1 & 22 \\
\hline
\end{tabular}

${ }^{a}$ Events that were both observed and forecasted are hits, events that were observed but not forecasted are misses, events that were forecasted but not observed are false alarms, and events that were not forecasted and not observed are correct rejections. The analysis of the 36 data-model comparisons yielded a probability of detection (POD) of $92.9 \%$, a false alarm rate of $0 \%$, a critical success index (CSI) of $92.9 \%$, and a Heidke skill score (HSS) of 0.941 .

inner magnetosphere. Although the dynamics are lacking, the average warm plasma characteristics are captured reasonably well by the coupled codes. In stark contrast, the model plasma from the 2004 event (Figure 10) remains stagnant throughout. Neither the cold or warm populations are captured well, even though the in situ measurements show that the real plasma is equally unexciting. Because of this, plasma results are less dependable during periods of steadily northward IMF $B_{z}$.

[30] The observed plasma distribution in Figure 9 exhibits a feature that may exacerbate the discrepancy between the measured and modeled integrated plasma properties. As warm electrons build up on the spacecraft's body, cold ions are accelerated toward the sensor, raising their measured energy. This generates a "cold ion line," as observed from 1600 UT to the end of the event. This draws cold particles into the warm plasma integration window $(>100 \mathrm{eV})$, causing the overall measured density to rise and temperature to fall. This effect cannot be properly removed from the MPA data at the present time.

\section{Summary of Results}

[31] Table 2 displays the average nRMSE and correlation coefficient scores for the data-model comparisons. They are divided up into three orbit categories: polar orbit (consisting of the Polar satellite), geosynchronous orbit (GOES satellites), and outer magnetosphere orbits (Geotail and a single Cluster satellite). For the outer magnetosphere category, any satellite that was upstream of the bow shock was discarded as it was merely mirroring the upstream solar wind input of the simulation. Performance measures were calculated for the three orthogonal components of the magnetic field in GSM coordinates as well as the total magnitude. The number of comparisons included in each average $(n)$ is also listed.

[32] Overall performance of the coupled models to predict magnetic field values was good. All average nRMSE scores were well below 1, showing that forecast values are closer to the measured values than the average magnitude of the measured quantity. Average correlation coefficients are greater than 0.6 for all components of the magnetic field, indicating that the model is trending with the data. In every case, the nRMSE for the total field strength was far lower than any of the components, implying that the field strength is represented well, while the field orientation is not as accurate. The opposite is true for the correlation coefficients, where correlation drops significantly for field magnitude (with the exception of the Polar satellite). This is because, at geosynchronous orbit, the field magnitude has less variation than the individual components, so smaller variations have a large effect on correlation.

[33] Performance of the coupled models decreases as grid resolution decreases (equivalently, as distance from the Earth increases.) The data-forecast comparisons using Polar data performed the best (Table 2), as the field is often dominated by the intrinsic dipole as the satellite passes close to the Earth. Correlation coefficients are especially strong (average of $>0.9$ ) for this orbit type. The framework does not perform as well at geosynchronous orbit (Table 2), but average nRMSE is still low $(<0.6)$ and there is a strong correlation for all three components of the magnetic field (average $>0.7$ ). Forecasts for satellites in the outer magnetosphere/sheath or downstream solar wind have the weakest performance, however all average nRMSE values remain well below one. The forecasts and data values are weakly correlated; coefficients for each component ranges from 0.607 to 0.666 .

[34] The particle comparison results are presented in Table 3. The second and third columns correspond to density and temperature results of $>100 \mathrm{eV}$ protons; the fourth and fifth columns correspond to $>30 \mathrm{eV}$ electron density and temperature. The first row is average nRMSE values, and the second row is average correlation coefficient. nRMSE values for the particle comparisons are near one, while the correlation coefficient results show that there is little to no correlation between the forecasts and data. While there is negligible difference between electron and proton density performance, on average the predicted proton temperature is more accurate than the electron temperature. Initial investigations found no apparent correlation between magnetic field performance and particle density or temperature performance.

[35] The results of the magnetopause crossing binary event study are presented in Tables 4 and 5 . Each entry in Tables 4 and 5 represents one evaluation of a time window from a single satellite's data-model comparison. For example, in Figure 4, a magnetopause crossing was both observed and predicted. For the whole-event time scale,

Table 5. Contingency Table for Magnetopause Crossings Using Hourly Time Resolution ${ }^{a}$

\begin{tabular}{ccc}
\hline & \multicolumn{2}{c}{ Observation } \\
\cline { 2 - 3 } Forecast & Yes & No \\
\hline Yes & 32 & 6 \\
No & 31 & 412 \\
\hline
\end{tabular}

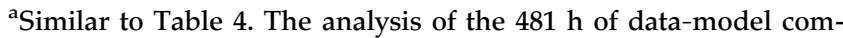
parisons yielded a POD of $50.8 \%$, a false alarm rate of $1.4 \%$, a CSI of $46.4 \%$, and HSS of 0.594 . 
this would add one to the "yes-yes" cell of Table 4 . For the hourly time scale, the first hour (0200 UT to 0300 UT) of Figure 4 would add one to the true negative ("no-no") cell, the fifth hour (0600 UT to 0700 UT) would increase the number in the miss ("yes-no") cell by one. This datamodel comparison would contribute only one value to Table 4 and twelve values to Table 5 .

[36] When examining magnetopause crossings on a whole-event time scale (Table 4), the SWMF yields a very high POD and CSI (both 92.9\%) and produced zero false alarms in all 36 satellite data-model pairs examined in the 10 event study. The HSS is 0.941 , a nearly perfect score indicating a high improvement in skill compared to a random forecast. Increasing the time resolution to hourly (Table 5) nearly halved the POD (50.8\%) and CSI $(46.4 \%)$. HSS drops to 0.594 , indicating a loss of skill for this time resolution but still a substantial increase compared to a random forecast. The false alarm rate rose only a negligible amount, from $0 \%$ to $1.4 \%$. The low false alarm rates show that the SWMF can provide value as a magnetopause crossing predictor for geosynchronous satellites because it rarely predicts a crossing that does not occur. The number of missed crossings, however, is still an issue that needs to be addressed.

\section{Conclusions}

[37] This study demonstrates the capabilities of the SWMF to predict satellite-specific magnetic field, plasma energy distribution, and geosynchronous magnetopause crossings. The model predicts large-scale magnetic perturbations reasonably well, even in regions of low resolution. Integrated plasma density and temperature values predicted by the model are poor. All simulations were completed at near real time run speeds using a limited amount of computing resources, demonstrating the SWMF's operational capabilities.

[38] The coupled codes also proved competent at predicting magnetopause crossings for satellites in geosynchronous orbits. The exceptionally low false alarm rates provide operational users confidence that a modeled crossing nearly always correctly predicts a real crossing. Missed crossings may still be a problem, especially those driven by solar wind pressure pulses. These results must be expanded upon in the future by investigating the crossings with finer time resolution and applying a full signal processing analysis to the binary results as outlined by Jolliffe and Stephenson [2003].

[39] Several deficiencies need to be addressed in order to improve the results presented here. The most important problems are the large magnetic reconnection rates, under stretched field lines in the inner magnetosphere, and lack of cold particle sources in the inner magnetosphere. These issues may be treated by improving and expanding upon the physics used in the various models. For example, a nonideal version of the BATSRUS MHD code that includes the Hall resistive term in the induction equation [Tóth et al., 2008] could be used in place of the version used here in order to improve reconnection physics. More SWMF components can be added as well, such as the Polar Wind Outflow Model [Glocer et al., 2007], which would act as a cold plasma source for the inner magnetosphere. Such changes may improve the nRMSE and correlation coefficient scores, but require additional computational resources.

[40] Future work will also include comparisons between SWMF predictions and rudimentary prediction methods and computationally inexpensive empirical codes. Such comparisons test for "skill," or the improvement in quality of one prediction method over another. Such work quantifies the advantage of using the computationally expensive SWMF over other predictive methods.

[41] The results presented here will serve as a baseline to compare to future validation results of similar quantities. This allows model developers to track code performance as the system evolves, and observe the impact new codes have on the coupled system. It also provides operational users with quantitative assessment of the system's performance. This and future studies are vital for scientific and operational users of the SWMF, who need to fully understand the model's capabilities.

[42] Acknowledgments. The authors would like to thank Michelle Thomsen from Los Alamos National Laboratory and Richard Wolf and Stanislav Sazykin for their input. We acknowledge the use of data from the ACE, Wind, Geotail, Cluster, GOES, and LANL Geosynchronous satellite missions. This work was supported by grants from the NSF (grants ATM0639336 and ATM0325332) and AFOSR (grant FA955007-1-0434).

\section{References}

Baker, D. (2000), The occurrence of operational anomalies in spacecraft and their relationship to space weather, IEEE Trans. Plasma Sci., 28, 2007-2016.

De Zeeuw, D. L., T. I. Gombosi, C. P. T. Groth, K. G. Powell, and Q. F. Stout (2000), An adaptive MHD method for global space weather simulations, IEEE Trans. Plasma Sci., 28, 1956-1965.

De Zeeuw, D. L., S. Sazykin, R. A. Wolf, T. I. Gombosi, A. J. Ridley, and G. Tóth (2004), Coupling of a global MHD code and an inner magnetosphere model: Initial results, J. Geophys. Res., 109, A12219, doi:10.1029/2003JA010366.

Feynman, J., and S. Gabriel (2000), On space weather consequences and predictions, J. Geophys. Res., 105, 10,543-10,564, doi:10.1029/ 1999JA000141.

Glocer, A., T. I. Gombosi, G. Toth, K. C. Hansen, A. J. Ridley, and A. Nagy (2007), Polar wind outflow model: Saturn results, J. Geophys. Res., 112, A01304, doi:10.1029/2006JA011755.

Gombosi, T. I., G. Tóth, D. L. De Zeeuw, K. C. Hansen, K. Kabin, and K. G. Powell (2002), Semi-relativistic magnetohydrodynamics and physics-based convergence acceleration, J. Comput. Phys., 177, 176-205.

Harel, M., R. A. Wolf, P. H. Reiff, R. W. Spiro, W. J. Burke, F. J. Rich, and M. Smiddy (1981), Quantitative simulation of a magnetospheric substorm: 1. Model logic and overview, J. Geophys. Res., 86, 22172241.

Horne, R. (2001), Space weather parameters required by the users, Contractor Rep. WP1300 and WP1400, Eur. Space Agency, Paris.

Jolliffe, I., and D. Stephenson (2003), Forecast Verification: A Practitioner's Guide in Atmospheric Science, John Wiley, Hoboken, N. J.

Lyon, J. G., J. A. Fedder, and C. M. Mobarry (2004), The Lyon-FedderMobarry (LFM) global MHD magnetospheric simulation code, J. Atmos. Sol. Terr. Phys., 66, 1333-1350. 
McComas, D. J., S. J. Bame, B. L. Barraclough, J. R. Donart, R. C. Elphic, J. T. Gosling, M. B. Moldwin, K. R. Moore, and M. F. Thomsen (1993), Magnetospheric plasma analyzer: Initial three-spacecraft observations from geosynchronous orbit, J. Geophys. Res., 98, $13,453-13,465$.

McComas, D. J., R. C. Elphic, M. B. Moldwin, and M. F. Thomsen (1994), Plasma observations of magnetopause crossings at geosynchronous orbit, J. Geophys. Res., 99, 21,249-21,255.

Papitashvili, V. O., and F. J. Rich (2002), High-latitude ionospheric convection models derived from Defense Meteorological Satellite Program ion drift observations and parameterized by the interplanetary magnetic field strength and direction, J. Geophys. Res., 107(A8), 1198, doi:10.1029/2001JA000264.

Pirjola, R., K. Kauristie, H. Lappalainen, A. Viljanen, and A. Pulkkinen (2005), Space weather risk, Space Weather, 3, S02A02, doi:10.1029/ 2004 SW000112.

Powell, K., P. Roe, T. Linde, T. Gombosi, and D. L. De Zeeuw (1999), A solution-adaptive upwind scheme for ideal magnetohydrodynamics, J. Comp. Phys., 154, 284-309.

Raeder, J., R. L. McPherron, L. A. Frank, S. Kokubun, G. Lu, T. Mukai, W. R. Paterson, J. B. Sigwarth, H. J. Singer, and J. A. Slavin (2001), Global simulation of the Geospace Environment Modeling substorm challenge event, J. Geophys. Res., 106, 381-395.

Ridley, A. J. (2007), Alfvén wings at Earth's magnetosphere under strong interplanetary magnetic fields, Ann. Geophys., 25, 533-542.

Ridley, A. J., and M. W. Liemohn (2002), A model-derived storm time asymmetric ring current driven electric field description, J. Geophys. Res., 107(A8), 1151, doi:10.1029/2001JA000051.

Ridley, A. J., D. L. De Zeeuw, T. I. Gombosi, and K. G. Powell (2001), Using steady state MHD results to predict the global state of the magnetosphere-ionosphere system, J. Geophys. Res., 106, 30,06730,076 .

Ridley, A. J., K. C. Hansen, G. Tóth, D. L. De Zueew, T. I. Gombosi, and K. G. Powell (2002), University of Michigan MHD results of the Geospace Global Circulation Model metrics challenge, J. Geophys. Res., 107(A10), 1290, doi:10.1029/2001JA000253.

Ridley, A. J., T. I. Gombosi, and D. L. De Zeeuw (2004), Ionospheric control of the magnetosphere: Conductance, Ann. Geophys., 22, 567-584.

Rufenach, C. L., R. F. Martin Jr., and H. H. Sauer (1989), A study of geosynchronous magnetopause crossing, J. Geophys. Res., 94, $15,125-15,134$
Sazykin, S. Y. (2000), Theoretical studies of penetration of magnetospheric electric fields to the ionosphere, Ph.D. thesis, Utah State Univ., Logan.

Toffoletto, F., S. Sazykin, R. Spiro, and R. Wolf (2003), Inner magnetospheric modeling with the Rice Convection Model, Space Sci. Rev. 107, 175-196.

Tóth, G., et al. (2005), Space weather modeling framework: A new tool for the space science community, J. Geophys. Res., 110, A12226, doi:10.1029/2005JA011126.

Tóth, G., D. L. De Zeeuw, T. I. Gombosi, W. B. Manchester, A. J. Ridley, I. V. Sokolov, and I. I. Roussev (2007), Sun-to-thermosphere simulation of the 28-30 October 2003 storm with the Space Weather Modeling Framework, Space Weather, 5, S06003, doi:10.1029/ 2006 SW000272.

Tóth, G., Y. J. Ma, and T. I. Gombosi (2008), Hall magnetohydrodynamics on block adaptive grids, J. Comput. Phys., 227, 6967-6984, doi:10.1016/j.jcp.2008.04.010.

Tsyganenko, N. A. (2002a), A model of the near magnetosphere with a dawn-dusk asymmetry: 1 . Mathematical structure, J. Geophys. Res., 107(A8), 1179, doi:10.1029/2001JA000219.

Tsyganenko, N. A. (2002b), A model of the near magnetosphere with a dawn-dusk asymmetry: 2. Parameterization and fitting to observations, J. Geophys. Res., 107(A8), 1176, doi:10.1029/2001JA000220.

Wang, H., A. J. Ridley, and H. Lühr (2008), Validation of the Space Weather Modeling Framework using observations from CHAMP and DMSP, Space Weather, 6, S03001, doi:10.1029/2007SW000355.

Weimer, D. R. (1996), A flexible, IMF dependent model of high-latitude electric potential having "space weather" applications, Geophys. Res. Lett., 23, 2549-2552.

Wolf, R. A. (1983), The quasi-static (slow-flow) region of the magnetosphere, in Solar Terrestrial Physics, edited by R. L. Carovillano and J. M. Forbes, pp. 303-368, D. Reidel, Hingham, Mass.

Yu, Y., and A. J. Ridley (2008), Validation of the Space Weather Modeling Framework using ground-based magnetometers, Space Weather, 6, S05002, doi:10.1029/2007SW000345.

A. J. Ridley and D. T. Welling, Department of Atmospheric, Oceanic, and Space Sciences, University of Michigan, 2455 Hayward St., Ann Arbor, MI 48109, USA. (dwelling@umich.edu) 